\title{
T-box transcription factor Brachyury expression is correlated with epithelial-mesenchymal transition and lymph node metastasis in oral squamous cell carcinoma
}

\author{
IKUMI IMAJYO $^{1 *}$, TSUYOSHI SUGIURA $^{1 *}$, YOSUKE KOBAYASHI $^{1}$, MIYUKI SHIMODA $^{1}$, KOTARO ISHII $^{1}$, \\ NAONARI AKIMOTO $^{1}$, NAOYA YOSHIHAMA ${ }^{1}$, IEYOSHI KOBAYASHI ${ }^{2}$ and YOSHIHIDE MORI ${ }^{1}$ \\ ${ }^{1}$ Division of Maxillofacial Diagnostic and Surgical Sciences, Department of Oral and Maxillofacial Surgery, \\ ${ }^{2}$ Division of Maxillofacial Diagnostic and Surgical Sciences, Department of Oral Pathology, \\ Graduate School of Dental Science, Kyushu University, Higashi-ku, Fukuoka 812-8582, Japan
}

Received July 26, 2012; Accepted September 14, 2012

DOI: 10.3892/ijo.2012.1673

\begin{abstract}
The prognosis of patients with oral squamous cell carcinoma (SCC) is influenced by the presence of lymph node metastasis. Epithelial-mesenchymal transition (EMT), a process that involves events that convert adherent epithelial cells into individual migratory cells that can invade the extracellular matrix, is critical for cancer progression. Recently, the T-box transcription factor Brachyury was reported to promote EMT in human carcinoma cell lines. We analyzed the relationship between EMT (assessed by staining for E-cadherin and Vimentin) and the expression of Brachyury in association with lymph node metastasis in oral SCC. Oral SCC biopsy specimens (152 cases) were examined immunohistochemically for the expression of E-cadherin, Vimentin and Brachyury. Expression of Brachyury was correlated with EMT $(p=0.035)$ and was significantly associated with lymph node and distant metastasis $(\mathrm{p}<0.05)$. Logistic regression analysis showed that Brachyury and EMT were predictive factors for lymph node metastasis (odds ratio 4.390 and 5.936, respectively) and that EMT was a predictive factor for distant metastases (odds ratio 11.786). Our findings present clinical evidence for an important role of Brachyury in EMT in oral SCC, and suggest that Brachyury and EMT patterns are useful prognostic markers.
\end{abstract}

\section{Introduction}

Squamous cell carcinoma (SCC) is the most common malignant tumor of the oral cavity and head and neck. Oral SCC involves

Correspondence to: Dr Tsuyoshi Sugiura, Division of Maxillofacial Diagnostic and Surgical Sciences, Oral and Maxillofacial Surgery, Graduate School of Dental Science, Kyushu University, 3-1-1 Maidashi, Higashi-ku, Fukuoka 812-8582, Japan

E-mail: sugiura@dent.kyushu-u.ac.jp

${ }^{*}$ Contributed equally

Key words: epithelial-mesenchymal transition, Brachyury, squamous cell carcinoma, metastasis, lymph nodes lymphatic metastasis, but not blood-borne metastasis, and it metastasizes to the regional lymph nodes in $30-40 \%$ of cases. Patient prognosis depends on the presence of lymph node metastasis. However, little is known about the molecular mechanisms underlying lymph node metastasis in SCC of the oral cavity. We have previously reported the relationship between lymphangiogenesis and the expression of VEGF-C and VEGF-D in association with lymph node metastasis in oral SCC.

Epithelial-mesenchymal transition (EMT) refers to a series of events that results in conversion of epithelial cells which adhere each other to individual migratory cells that can invade into extracellular matrix (1). EMT is crucial for appropriate development in the early embryo, and this process also plays an important role in adults during wound healing, tissue regeneration, and cancer progression (2). It is closely related to poor prognosis of various types of cancer [gastric (3), colon (4), breast $(5,6)$, esophageal (7), lung (8), and ovarian (9)]. In oral cancer, EMT in tongue squamous cell carcinoma (SCC) has been reported to be related to tumor satellite formation (10) and lymph node metastasis (11). These reports suggest that EMT is a precursor of SCC metastasis and that it induces tumor cell dissemination from the primary tumor site.

The EMT events during tumor progression are controlled by genes that are normally expressed in the early embryo (e.g., Twist, Snail, Slug, Goosecoid and SIP1) (7,12-15). The transcription factors encoded by these genes can induce characteristics of mesenchymal cells, such as cell motility and invasiveness, in tumor cells. For example, the expression of Twist is increased in various types of malignant tumors, including breast cancer, prostate cancer, gastric cancer, and melanoma $(16,17)$.

Recently, the T-box transcription factor Brachyury, a gene required for mesoderm formation during early development $(18,19)$, was reported to promote EMT in human carcinoma cell lines (20). This study also showed that ectopic overexpression of Brachyury by gene transfection in human carcinoma cells induced characteristic changes of EMT. Based on these findings, it is proposed that EMT in cancer cells is controlled by mechanisms similar to EMT in human developmental processes.

In this study, we investigated the patterns and levels of expression of T-box transcription factor Brachyury, using immu- 
Table I. Classification of staining density.

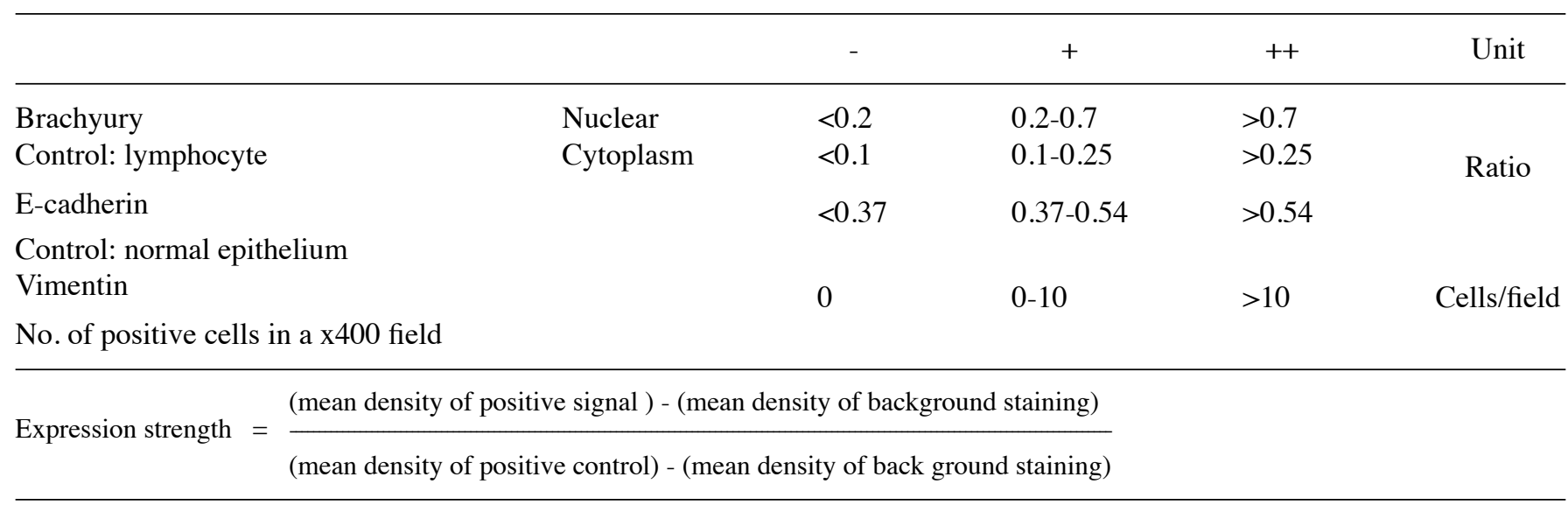

nohistochemistry on oral SCC specimens, and analyzed the relationship between Brachyury expression and EMT. We also statistically analyzed the correlation between Brachyury expression and prognosis, focusing on lymph node metastasis.

\section{Patients and methods}

Patients and tumor specimens. This study was based on a retrospective cohort of 152 patients who had been diagnosed with primary oral SCC and treated at the Department of Oral and Maxillofacial Surgery, Kyushu University Hospital, Fukuoka, Japan, between 1993 and 2006. All biopsies were obtained from the patients before any treatment was administered. The clinicopathological information associated with each case, including age, gender, tumor size, nodal status, location, treatment, and the presence or absence of recurrence and metastasis, was obtained from patient files. Ninety-seven men and 55 women were involved; median age was 61 years, range 24-85. All patients were staged according to the UICC TNM Classification of Malignant Tumors (21). When cervical metastasis was clinically equivocal, neck dissection was performed, and the metastases were diagnosed histologically. The protocol for the research project was approved by the appropriate Ethics Committee of Kyushu University.

Immunohistochemistry. Biopsy samples were fixed in $10 \%$ neutralized buffered-formalin. Consecutive $4-\mu \mathrm{m}$ sections were cut and deparaffinized with xylene, rehydrated in a graded alcohol series, and heat treated with Target Retrieval Solution (Dako, Carpinteria, CA, USA) before histopathological and immunohistochemical analysis. The grade of tumor differentiation was determined using the criteria proposed by the World Health Organization (22). The histological mode of invasion was classified according to the Anneroth classification (23).

To block endogenous peroxide activity, $3 \% \mathrm{H}_{2} \mathrm{O}_{2}$ was applied, and non-specific antibody binding was blocked with $10 \%$ normal serum in Tris- $\mathrm{HCl}$ buffer. The sections were incubated overnight at $4^{\circ} \mathrm{C}$ with the following primary antibodies: rabbit polyclonal antibody against human Brachyury (H-210, Santa Cruz Biotechnology, Santa Cruz, CA, USA); mouse monoclonal antibody against human E-cadherin (610181, BD Bioscience, San Jose, CA, USA); and goat polyclonal antibody against human Vimentin (C-20, Santa Cruz Biotechnology, Santa Cruz, CA, USA). Immunostaining was performed with the Histofine SAB-PO kit (Nichirei, Tokyo, Japan), according to the manufacturer's instructions. The immunolocalization of the protein was visualized using a DAB substrate kit (Nichirei). Sections were counterstained with $0.5 \%$ hematoxylin, dehydrated, cleared, and mounted. Negative control staining involved substituting nonimmune goat serum for the primary antibodies.

We evaluated the staining pattern of the indicated proteins at the invasive edge of the primary tumors for all the specimens. The density of staining for Brachyury and E-cadherin was evaluated by measuring the difference between the mean density (pixels) of 10 randomly selected positive-staining fields in SCC and the mean density of background staining, using the analyze tool in Photoshop CS5 Extended (Adobe Systems Inc., San Jose, CA, USA). To avoid any measurement errors derived from staining heterogeneity, we also measured the mean density of staining for each protein in normal cells (lymphocytes for Brachyury and normal epithelium for E-cadherin) as positive controls in the same sample, and the ratio of staining density between SCC and positive control was calculated. This ratio was taken to represent the expression level of the protein. For Vimentin, the number of the positive SCC cells in a low-power field was counted. The results were classified into 3 groups for each protein as shown in Table I: ++, strong staining; +, moderate staining; and -, negative (Figs. 1 and 2). Two independent observers blinded to the patient status scored the samples.

Immunofluorescence staining. Sections were cut and mounted as described above and heat treated with Target Retrieval Solution (Dako) before immunofluorescence staining. Non-specific antibody binding was blocked with 3\% BSA in phosphate-buffered saline (PBS) for $1 \mathrm{~h}$ at room temperature. The sections were incubated overnight at $4^{\circ} \mathrm{C}$ with primary antibodies as described above. Following three 10-min washes in PBS, sections were incubated with secondary antibodies for $1 \mathrm{~h}$ at room temperature. The secondary antibodies (Invitrogen, Carlsbad, CA, USA) used were: for Brachyury, Alexa Fluor 498 goat anti-mouse, catalog no. A11034; for E-cadherin, Alexa Fluor 350 goat anti-mouse, catalog no. A11029; for Vimentin, Alexa Fluor 594 donkey anti-goat, catalog no. A11058. After additional washes, images were captured using a Z-axis-controlled microscope 

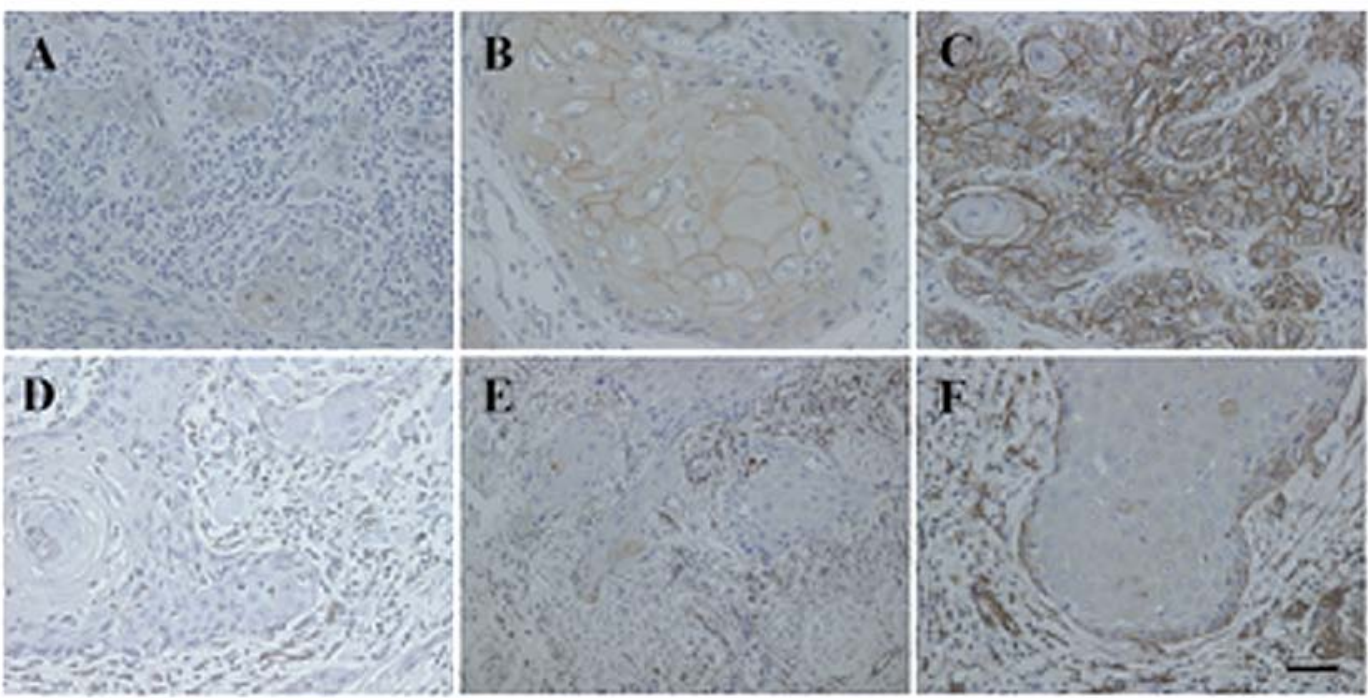

Figure 1. Immunohistochemical analysis of E-cadherin and Vimentin expression in SCC tissue. For the histopathological and immunohistochemical analysis 4- $\mu \mathrm{m}$-thick sections were used as described in Patients and methods. Staining intensity of E-cadherin and Vimentin in SCC cells was classified as follows: ++ , strong staining; +, moderate staining; -, negative staining as described in Table I. Photomicrographs show representative tissue staining patterns of each group. (A-C) E-cadherin; (D-F) Vimentin; (A and D) (-); (B and E) (+); (C and F) (++). Bar, $50 \mu \mathrm{m}$.

\section{Type I}
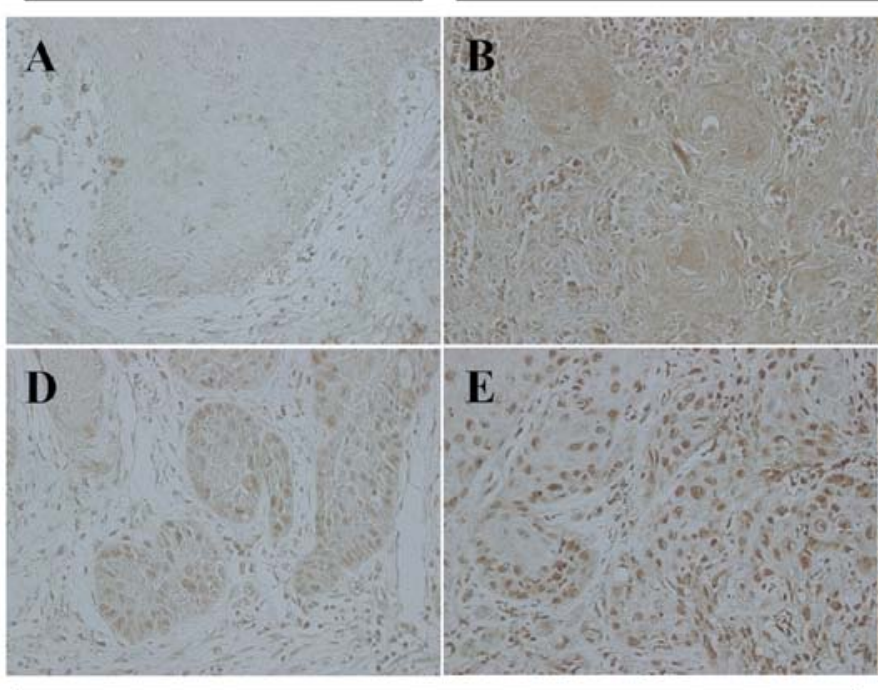

TypeIII

\section{Type II}
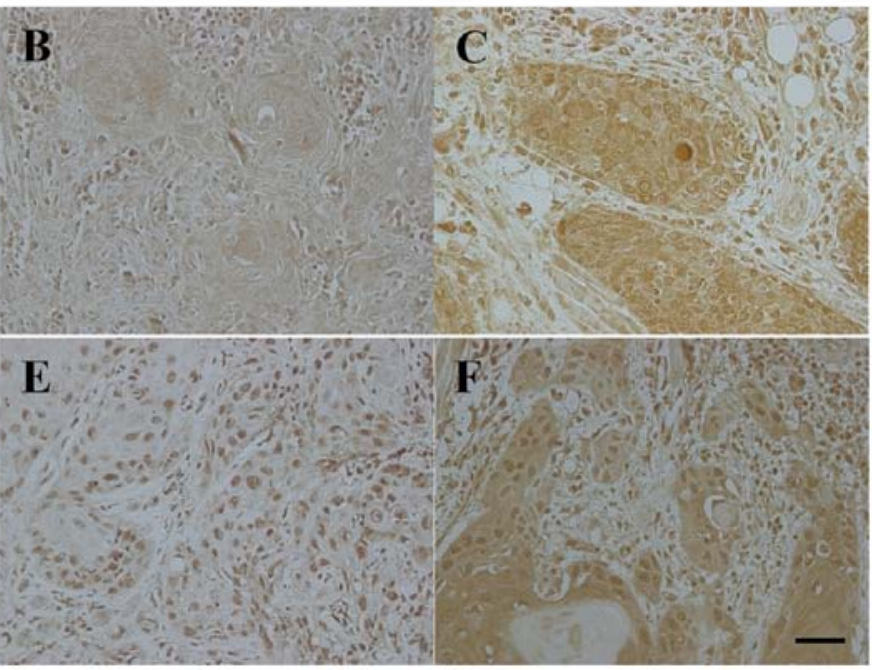

TypeIV

Figure 2. Immunohistochemical analysis of Brachyury expression in SCC tissue. Staining intensity of Brachyury in SCC cells was classified as follows: ++ , strong staining; +, moderate staining; and -, negative staining as described in Table I. Pattern of cellular localization was scored as: Type I, negative staining; Type II, positive only in the cytoplasm; Type III, positive only in the nucleus; Type IV, positive in both the cytoplasm and nucleus. Photomicrographs show representative staining patterns of each group. (A) Negative (-); (B) Weak positive in the cytoplasm (+); (C) Strong positive in the cytoplasm (++); (D) Weak positive in the nucleus $(+)$; (E) Strong positive in the nucleus (++); and (F) Strong positive in the cytoplasm and nucleus (++). Bar, $50 \mu \mathrm{m}$.

with a CCD camera (BZ-8000; Keyence, Osaka, Japan) and processed for deconvoluted fluorescence imaging.

Statistical analysis. Statistical analysis was performed with the statistical software package SPSS for Windows (Abacus Concepts, Berkeley, CA, USA). Relationships between staining intensity of markers and various clinicopathological factors were assessed with the $\chi^{2}$ test. To define independent risk factors for lymph node metastasis and distant metastasis, univariate and multivariate analysis were performed with a logistic regression analysis. The survival rate was estimated with the Kaplan-Meier method and analyzed using the logrank test. Differences were considered significant at $\mathrm{p}<0.05$.

\section{Results}

EMT pattern in oral SCC. We examined EMT in oral SCC lesions by assessing loss of E-cadherin and gain of Vimentin using immunohistochemistry. E-cadherin was localized on the cell membrane of SCC cells but was lost in some cases, 
Table II. Relation between EMT and clinicopathological factors.

\begin{tabular}{|c|c|c|c|c|c|c|c|c|c|c|c|c|}
\hline & \multirow[b]{2}{*}{ Cases } & \multicolumn{4}{|c|}{ E-cadherin } & \multicolumn{4}{|c|}{ Vimentin } & \multicolumn{3}{|c|}{ EMT } \\
\hline & & - & + & ++ & p-value & - & + & ++ & p-value & - & + & p-value \\
\hline \multicolumn{13}{|l|}{ Age } \\
\hline$\leq 65$ & 88 & 30 & 28 & 30 & NS & 76 & 8 & 4 & NS & 83 & 5 & NS \\
\hline$>65$ & 64 & 18 & 28 & 18 & & 48 & 9 & 7 & & 57 & 7 & \\
\hline \multicolumn{13}{|l|}{ Sex } \\
\hline Male & 97 & 29 & 33 & 35 & NS & 78 & 12 & 7 & NS & 90 & 7 & NS \\
\hline Female & 55 & 19 & 23 & 13 & & 46 & 5 & 4 & & 50 & 5 & \\
\hline \multicolumn{13}{|l|}{ Clinical stage } \\
\hline $\mathrm{T} 1$ & 37 & 13 & 13 & 11 & NS & 33 & 3 & 1 & NS & 36 & 1 & NS \\
\hline $\mathrm{T} 2$ & 62 & 17 & 25 & 20 & & 50 & 8 & 4 & & 58 & 4 & \\
\hline T3 & 29 & 12 & 9 & 8 & & 25 & 3 & 1 & & 25 & 4 & \\
\hline $\mathrm{T} 4$ & 24 & 6 & 9 & 9 & & 16 & 3 & 5 & & 21 & 3 & \\
\hline \multicolumn{13}{|l|}{ Tumor site } \\
\hline Buccal mucosa & 14 & 7 & 5 & 2 & NS & 10 & 3 & 1 & 0.021 & 13 & 1 & NS \\
\hline Upper gingiva & 7 & 2 & 4 & 1 & & 3 & 1 & 3 & & 5 & 2 & \\
\hline Lower gingiva & 39 & 10 & 13 & 16 & & 34 & 3 & 2 & & 36 & 3 & \\
\hline Tongue & 81 & 27 & 26 & 28 & & 67 & 10 & 4 & & 75 & 6 & \\
\hline Oral floor & 11 & 2 & 8 & 1 & & 10 & 0 & 1 & & 11 & 0 & \\
\hline \multicolumn{13}{|c|}{ Lymph node involvement } \\
\hline Positive & 56 & 24 & 15 & 17 & 0.049 & 39 & 9 & 8 & 0.009 & 47 & 9 & 0.009 \\
\hline Negative & 96 & 24 & 41 & 31 & & 85 & 8 & 3 & & 93 & 3 & \\
\hline \multicolumn{13}{|l|}{ Distant metastases } \\
\hline Positive & 13 & 10 & 0 & 3 & 0.001 & 7 & 2 & 4 & 0.002 & 8 & 5 & 0.001 \\
\hline Negative & 139 & 38 & 56 & 45 & & 117 & 15 & 7 & & 132 & 7 & \\
\hline \multicolumn{13}{|c|}{ Tumor differentiation } \\
\hline Well & 132 & 37 & 50 & 45 & 0.041 & 107 & 15 & 10 & NS & 123 & 9 & NS \\
\hline Moderate & 19 & 11 & 6 & 2 & & 16 & 2 & 1 & & 16 & 3 & \\
\hline Poor & 1 & 0 & 0 & 1 & & 1 & 0 & 0 & & 1 & 0 & \\
\hline \multicolumn{13}{|l|}{ Pattern of invasion } \\
\hline 1 & 2 & 1 & 1 & 0 & NS & 2 & 0 & 0 & 0.030 & 2 & 0 & NS \\
\hline 2 & 37 & 10 & 14 & 13 & & 32 & 3 & 2 & & 35 & 2 & \\
\hline 3 & 82 & 23 & 31 & 28 & & 69 & 5 & 8 & & 77 & 5 & \\
\hline 4 & 31 & 14 & 10 & 7 & & 21 & 9 & 1 & & 26 & 5 & \\
\hline
\end{tabular}

$\chi^{2}$ test: NS, not significant. $\mathrm{p}<0.05$.

whereas Vimentin was stained in the cytoplasm of SCC cells and in the stromal tissue (Fig. 1). We categorized E-cadherin and Vimentin staining by staining intensity and scored the staining patterns as EMT (E-cadherin-negative, Vimentinpositive) or not EMT.

Table II summarizes clinicopathological data of all cases and E-cadherin and Vimentin immunoreactivity. The relationship between E-cadherin and Vimentin expression and clinicopathological factors was analyzed with the $\chi^{2}$ test. The staining intensity of E-cadherin on SCC cells was significantly associated with lymph node involvement $(\mathrm{p}=0.049)$, distant metastasis $(p=0.001)$, and tumor differentiation $(p=0.041)$, while the intensity of Vimentin staining was associated with lymph node involvement $(\mathrm{p}=0.009)$ and distant metastasis $(\mathrm{p}=0.002)$. The relationship between EMT pattern and clinicopathological factors was also analyzed with the $\chi^{2}$ test. Lymph node involvement $(\mathrm{p}=0.009)$ and distant metastasis $(\mathrm{p}=0.001)$ were significantly correlated with presence of the EMT pattern.

Pattern and level of expression of Brachyury in oral SCC. We next examined the expression of Brachyury in oral SCC lesions using immunohistochemistry. Brachyury protein was localized 

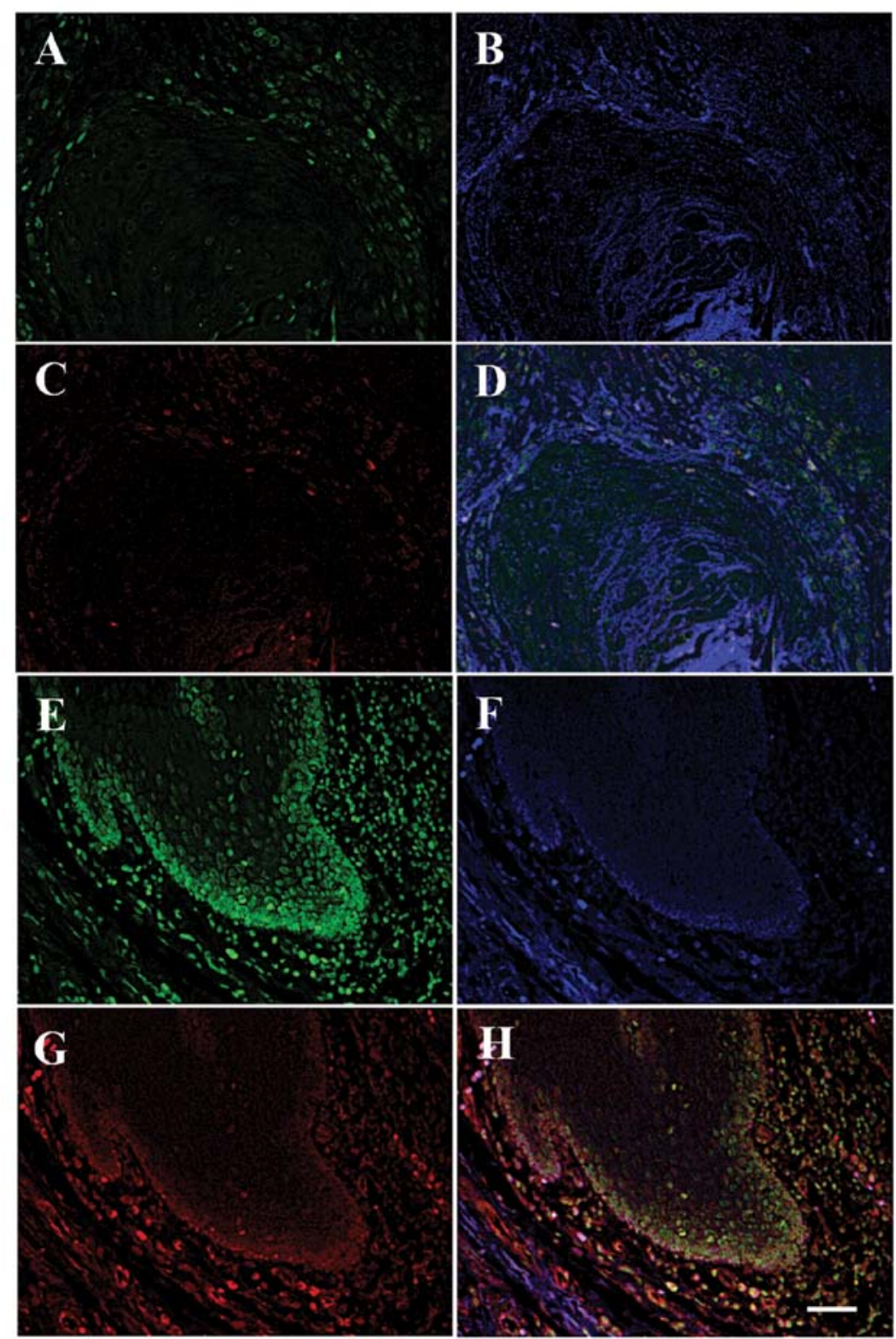

Figure 3. Triple immunofluorescence staining analysis of Brachyury, E-cadherin, and Vimentin expression in SCC tissue. Each protein was visualized by secondary antibodies: Brachyury, green; E-cadherin, blue; and Vimentin, red. (A-D) Representative staining of EMT-negative, Brachyury-negative SCC. (E-H) Representative staining of EMT-positive, Brachyury-positive SCC. (A and E) Brachyury; (B and F) E-cadherin; (C and G) Vimentin; and (D and H) Merged image. Bar, $50 \mu \mathrm{m}$.

in the cytoplasm and/or the nucleus of SCC cells. Brachyury was occasionally detected in lymphocytes around the SCC nests and in stromal cells surrounding the SCC nests. SCC cells in the invasive front tended to be stained strongly (Fig. 2). We categorized Brachyury staining by staining intensity in the cytoplasm or nucleus and by pattern of cellular localization: Type I, negative staining; Type II, positive only in the cytoplasm; Type III, positive only in the nucleus; and Type IV, positive in both the cytoplasm and nucleus. The relationship between Brachyury expression (intensity and pattern) and clinicopathological factors was analyzed with the $\chi^{2}$ test (Table III).

The rate of positive expression of Brachyury was $71.0 \%$ (Type I, 28.9\%; Type II, 11.8\%; Type III, 27.0\%; and Type IV, $32.2 \%$ ). Nuclear staining intensity of Brachyury in SCC cells was significantly associated with clinical $\mathrm{T}$ stage $(\mathrm{p}=0.001)$, lymph node involvement $(\mathrm{p}=0.003)$, tumor differentiation $(\mathrm{p}=0.043)$, and the pattern of invasion $(\mathrm{p}=0.024)$, while cytoplasmic intensity of Brachyury was associated only with lymph node involvement $(\mathrm{p}=0.028)$ and the pattern of invasion $(\mathrm{p}=0.022)$.

The staining pattern of Brachyury on SCC cells was more significantly associated with clinicopathological factors than intensity of Brachyury; the pattern was associated with clinical T stage $(p=0.001)$, lymph node involvement $(p=0.004)$, tumor differentiation $(\mathrm{p}=0.03)$, and the pattern of invasion $(\mathrm{p}=0.006)$.

Molecular localization of Brachyury and EMT markers in oral SCC. To find direct evidence that Brachyury is associated with EMT, we analyzed localization of Brachyury, E-cadherin, and Vimentin in oral SCC tissues by triple immunofluorescence staining. Fig. 3A shows that Brachyury-negative cells strongly express E-cadherin. In contrast, stromal cells express 


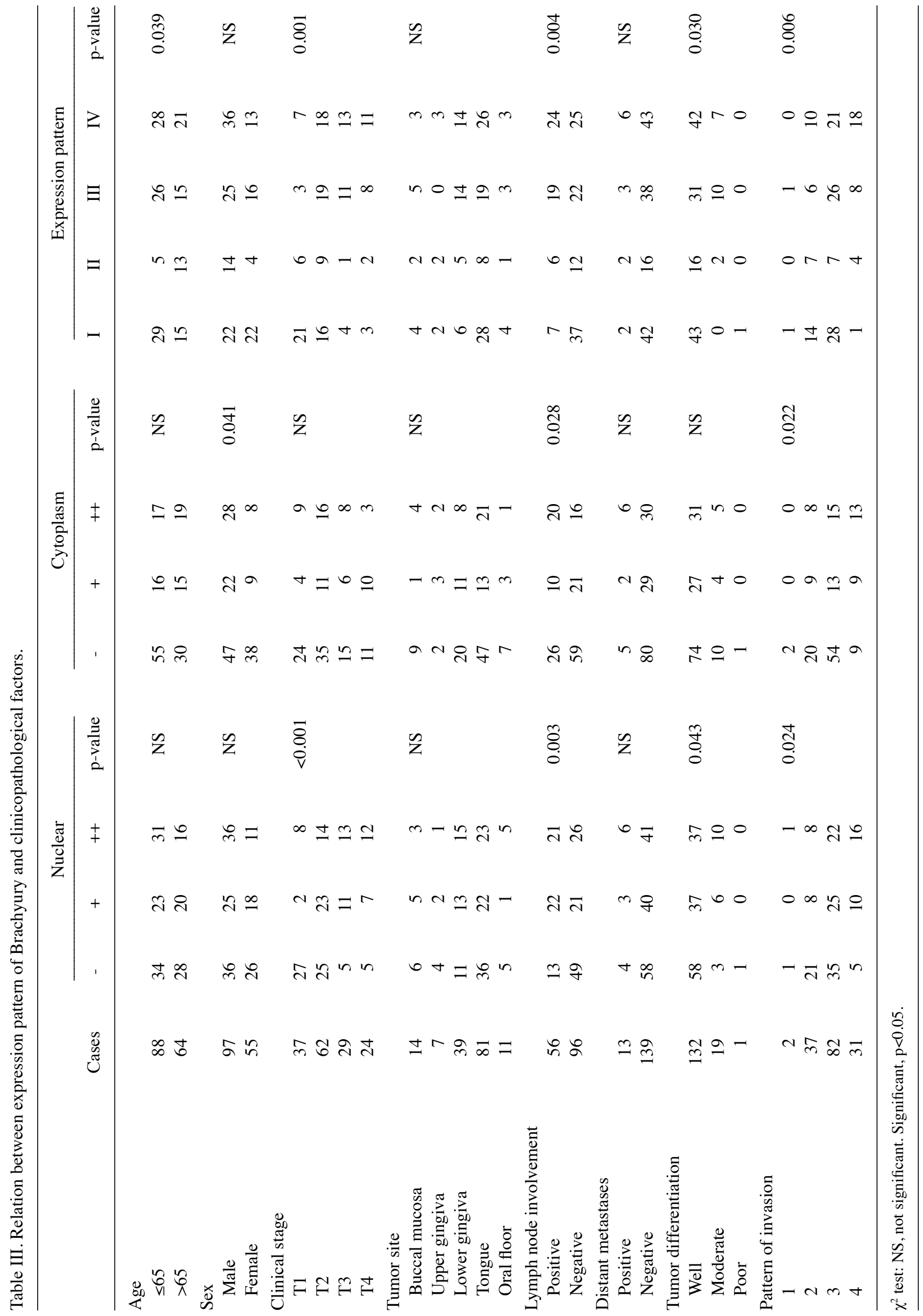


Table IV. Relationships among Brachyury expression, EMT markers, and EMT.

\begin{tabular}{|c|c|c|c|c|c|c|c|c|c|c|c|}
\hline \multirow[b]{2}{*}{$\begin{array}{l}\text { Brachyury expression } \\
\text { pattern }\end{array}$} & \multicolumn{4}{|c|}{ E-cadherin } & \multicolumn{4}{|c|}{ Vimentin } & \multicolumn{3}{|c|}{ EMT } \\
\hline & - & + & ++ & p-value & - & + & ++ & p-value & Negative & Positive & p-value \\
\hline I & 14 & 16 & 14 & NS & 42 & 1 & 1 & 0.002 & 43 & 1 & 0.035 \\
\hline II & 4 & 8 & 6 & & 11 & 4 & 3 & & 16 & 2 & \\
\hline III & 15 & 18 & 8 & & 38 & 2 & 1 & & 40 & 1 & \\
\hline IV & 15 & 14 & 20 & & 33 & 10 & 6 & & 41 & 8 & \\
\hline
\end{tabular}

$\chi^{2}$ test: NS, not significant. $\mathrm{p}<0.05$.

Table V. Logistic regression analysis of predictive factors.

\begin{tabular}{|c|c|c|c|c|c|c|}
\hline & \multicolumn{3}{|c|}{ Univariate analysis } & \multicolumn{3}{|c|}{ Multivariate analysis } \\
\hline & Odds ratio & p-value & $95 \% \mathrm{CI}$ & Odds ratio & p-value & $95 \% \mathrm{CI}$ \\
\hline \multirow{2}{*}{\multicolumn{7}{|c|}{$\begin{array}{l}\text { Lymph node involvement } \\
\text { Brachyury }\end{array}$}} \\
\hline & & & & & & \\
\hline Negative vs. positive & 4.39 & 0.001 & $1.799-10.714$ & 3.952 & 0.040 & $1.563-9.988$ \\
\hline \multicolumn{7}{|l|}{ E-cadherin } \\
\hline Negative vs. positive & 0.444 & 0.024 & $0.220-0.897$ & 0.478 & 0.089 & $0.204-1.120$ \\
\hline \multicolumn{7}{|l|}{ Vimentin } \\
\hline Negative vs. positive & 3.368 & 0.005 & $1.443-7.864$ & 2.020 & 0.215 & $0.665-6.131$ \\
\hline \multicolumn{7}{|l|}{ EMT } \\
\hline Negative vs. positive & 5.936 & 0.010 & $1.534-22.965$ & 1.533 & 0.653 & $0.238-9.890$ \\
\hline \multicolumn{7}{|l|}{ Distant metastases } \\
\hline \multicolumn{7}{|l|}{ Brachyury } \\
\hline Negative vs. positive & 2.381 & 0.272 & $0.506-11.215$ & 1.836 & 0.475 & $0.347-9.713$ \\
\hline \multicolumn{7}{|l|}{ E-cadherin } \\
\hline Negative vs. positive & 0.113 & 0.001 & $0.029-0.432$ & 0.141 & 0.023 & $0.026-0.766$ \\
\hline \multicolumn{7}{|l|}{ Vimentin } \\
\hline Negative vs. positive & 4.558 & 0.012 & $1.398-14.860$ & 2.506 & 0.468 & $0.210-29.969$ \\
\hline \multicolumn{7}{|l|}{ EMT } \\
\hline Negative vs. positive & 11.786 & $<0.001$ & $3.051-45.527$ & 1.533 & 0.771 & $0.086-27.307$ \\
\hline
\end{tabular}

Brachyury and Vimentin. Fig. 3B shows that SCC cells expressing both Brachyury and Vimentin were found at the invasive front of the primary nest.

Relationships between Brachyury expression and EMT in oral $S C C$. The relationship between Brachyury expression pattern and EMT (staining intensity of E-cadherin and Vimentin or scored pattern) was analyzed with the $\chi^{2}$ test (Table IV).

Brachyury expression pattern was significantly correlated with Vimentin expression $(\mathrm{p}=0.002)$. In particular, Type IV staining was strongly associated with expression of Vimentin. Brachyury expression pattern was also significantly correlated with $\operatorname{EMT}(p=0.035)$.

Logistic regression analysis of the predictive factors for lymph node and distant metastasis. To examine the significance of the predictive factors for lymph node metastasis, a logistic regression analysis was performed (Table V). A univariate analysis showed that EMT, positive expression of Brachyury, and Vimentin, and negative expression of E-cadherin were related to lymph node metastasis (odds ratios 5.936, 4.39, 3.368, and 0.444, respectively), while EMT, lacking expression of E-cadherin, and Vimentin were related to distant metastasis (odds ratios $11.768,0.113$, and 4.558 , respectively). Multivariate analysis also showed that positive expression of Brachyury was related to lymph node metastasis (odds ratio 3.952), while negative expression of E-cadherin was related to distant metastasis (odds ratio 0.141). Brachyury expression exhibited the highest odds ratios (4.390 and 3.952) among the predictive factors for lymph node metastasis, and E-cadherin exhibited the most significant odds ratios (0.113 and 0.141$)$ among the predictive factors for distant metastasis. 

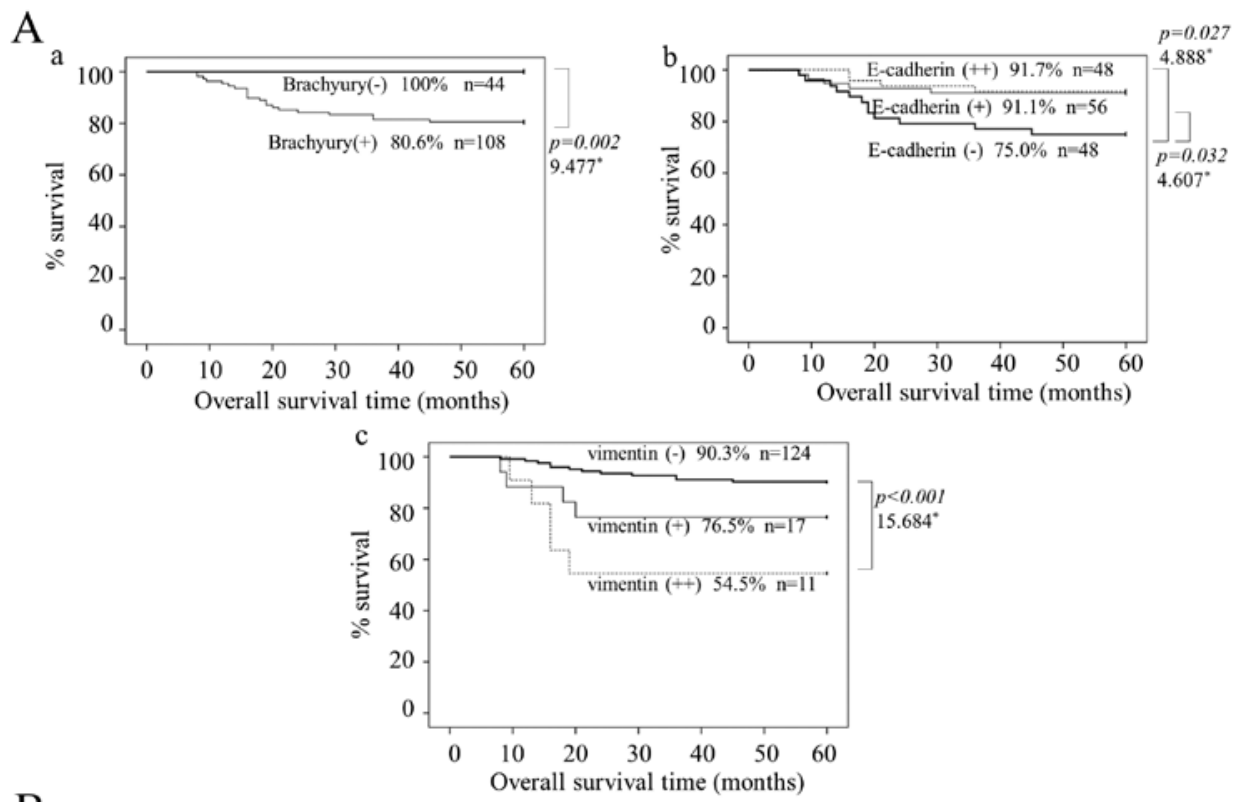

B
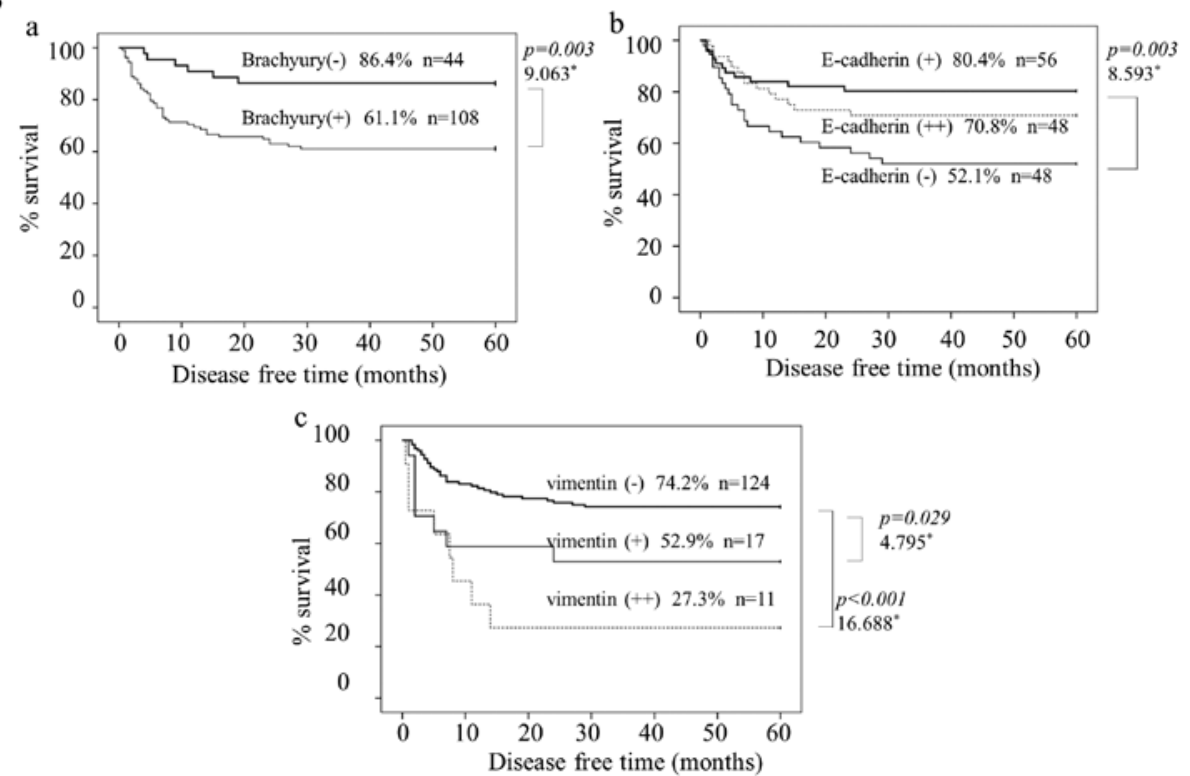

Figure 4. Kaplan-Meier survival analysis. Survival rate was estimated with the Kaplan-Meier method and analyzed using the log-rank test. Overall (A) and disease-free (B) survival of patients with tumors of the indicated status for (a) Brachyury, (b) E-cadherin, and (c) Vimentin are shown. ${ }^{*}{ }^{2}$ statistics.

Correlation between Brachyury expression and/or EMT and survival time. To investigate whether Brachyury expression and/or EMT pattern in biopsy specimens predicts outcome in patients with oral SCC, Kaplan-Meier analysis for staining intensity of Brachyury, E-cadherin, and Vimentin of the overall (Fig. 4A) and disease-free (Fig. 4B) survival times was performed (Fig. 4). The 5-year overall survival rate of patients with Brachyury-positive SCC (80.6\%) was significantly less than that of the negative counterpart $\left(100 \%, \log\right.$-rank test, $\left.\mathrm{p}=0.002 ; \chi^{2}, 9.477\right)$. The survival time of patients with E-cadherin-negative SCC (-) was significantly less than that of the weak $(+)$ and strong $(++)$ positive counterparts (log-rank test, $\mathrm{p}=0.032 ; \chi^{2} 4.607 ; \mathrm{p}=0.027 ; \chi^{2}$, 4.888 , respectively). The survival time of patients with strong
Vimentin-positive (++) SCC was significantly less than that of the negative counterpart (log-rank test, $\mathrm{p}<0.001 ; \chi^{2}, 15.684$ ).

We also analyzed the survival time for EMT pattern or Brachyury expression with EMT pattern by the KaplanMeier method (Fig. 5A, overall survival rate; Fig. 5B, disease-free survival rate). The survival time of patients with SCC exhibiting the EMT pattern was strikingly shorter than in EMT-negative groups. The 5-year overall survival rate in these patients was $41.7 \%$ (log-rank test, $\mathrm{p}=0.001$; $\left.\chi^{2}, 31.196\right)$ and the 5 -year disease-free survival rate was $25.0 \%$ (log-rank test, $\left.\mathrm{p}<0.001 ; \chi^{2}, 20.879\right)$. Moreover, survival time of patients with Brachyury expression and EMT pattern was significantly shorter than in Brachyurynegative groups; 5-year overall survival rate was $36.2 \%$ 

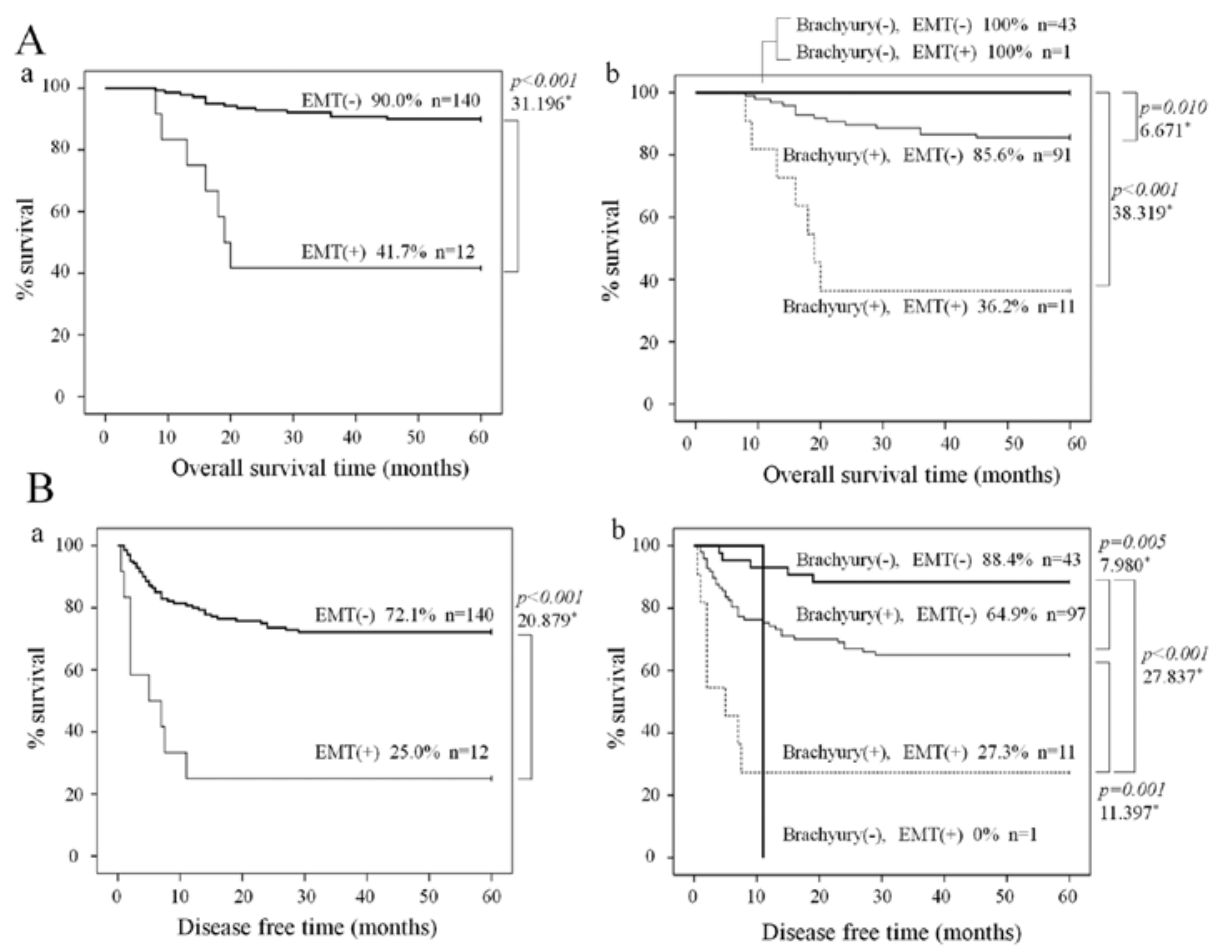

Figure 5. Kaplan-Meier survival analysis for EMT pattern and Brachyury expression. Survival rate was estimated with the Kaplan-Meier method and analyzed using the log-rank test. Overall (A) and disease-free (B) survival of patients with tumors of the indicated status for (a) EMT and (b) Brachyury with EMT are shown. ${ }^{*} \chi^{2}$ statistics.

(log-rank test, $\left.\mathrm{p}=0.01 ; \chi^{2}, 38.319\right)$ and 5-year disease-free survival rate was $27.3 \%$ (log-rank test, $\mathrm{p}<0.001 ; \chi^{2}, 27.837$ ).

\section{Discussion}

Cancer invasion and metastasis are crucial events in disease progression and largely determine prognosis of cancer patients. Many factors regulate cancer cell behavior, and correlation between clinical prognoses and expression of these factors in various tumors has led to their use as prognostic markers. Recently, EMT has been identified as not a factor but a crucial event in cancer invasion and metastasis during which many factors are simultaneously regulated $(24,25)$. In this regard, EMT patterns in tumor tissue are likely to be useful for estimating cancer prognosis.

We used loss of E-cadherin and gain of Vimentin as a marker of EMT, and we found a significant correlation between EMT pattern and lymph node involvement or distant metastasis (Table II). Interestingly, Vimentin expression showed a stronger relationship with distant metastasis than did E-cadherin expression. Loss of E-cadherin is thought to be a hallmark of EMT, because loss of E-cadherin loosens cell-cell adhesion enabling cancer cells to escape from the primary cancer nest (26). For example, in one recent study, the downregulation of E-cadherin shows significant difference compared to the pattern of invasion, tumor satellite formation, and tumor satellite size (10). On the other hand, Vimentin overexpression in cancer tissues also correlates with tumor growth, tumor invasion, and poor clinical outcome $(3,27)$. In oral cancer, expression of Vimentin significantly increases in groups stratified by tumor satellite distance (10).
Vimentin overexpression induces tyrosine kinase expression and results in cancer cell migration (28). This could explain the strong correlation between Vimentin expression and distant metastasis.

The mechanisms by which EMT is regulated in cancer cells are still not well understood. Recently, Fernando et al (20) reported that Brachyury, a gene required for mesoderm formation during early development, promotes EMT in human carcinoma cell lines. Their study showed that overexpression of Brachyury in human cancer cells induced characteristic changes of EMT, including elevated level of mesenchymal markers, decrease of epithelial markers, and increased cell migration and invasion. In our immunohistochemical analysis, Brachyury expressing cancer cells lost E-cadherin and gained Vimentin expression, consistent with previous reports and Fig. 3. Based on these data, we hypothesized that Brachyury expression is linked to EMT and correlated with disease prognosis. Therefore, we analyzed the relationship between Brachyury expression and clinicopathological findings (Table III). Brachyury expression correlated with clinical T stage, lymph node involvement, tumor differentiation, and pattern of invasion. Importantly, cellular localization of Brachyury is more important factor than staining intensity. Brachyury is a T-box transcription factor that transduces cellular signals by translocation to the nucleus. Nuclear localization of Brachyury was significantly related to malignant phenotypes in oral cancer (Table III). A role for Brachyury in promotion of EMT is supported by our finding that Brachyury cellular localization was significantly correlated with Vimentin expression and EMT pattern (Table IV). 
It is also noteworthy that EMT and Brachyury expression in oral SCC showed a significant correlation with lymph node involvement (Table V). These findings suggest that Brachyury is one of the key elements in the control of EMT and that EMT is one of the most important events in the development of malignant phenotypes.

The existence of self-renewing, stem-like cells within tumors, called cancer stem cells (CSCs), has been proved. CSCs form a minor population in cancer cells within a tumor and are defined by their ability to establish new tumors. Hence, CSCs are also called 'tumor-initiating cells' (29). During cancer cell metastasis, which is often enabled by EMT (30), cancer cells which escape from primary site would require a self-renewal capability similar to that exhibited by stem cells in order to generate a new tumor in distant site. In this regard, the EMT process may also give a self-renewal capability to metastatic cancer cells. Indeed, emerging evidence of a direct interaction between EMT and CSCs has been reported $(20,31,32)$. Expression of SOX2, a member of the SOX (SRY-related high mobility group box) family, was recently shown to be significantly associated with poor prognosis in oral tongue SCC (33). SOX2 was originally characterized as an important regulator of the maintenance of embryonic stem cell pluripotency (34). It is possible that SOX2 can function to maintain CSCs.

Like SOX2, Brachyury is an important gene in embryonic development and induces EMT in early embryonic stages and in cancer cells (35). These similarities raise the possibility that Brachyury is also an important regulator of CSC maintenance. Notably, recent clinical evidence suggests that Brachyury regulates CSCs in colorectal cancer (36). The report suggests that Brachyury regulates Nanog in mesenchymal-like cancer cells to impose a plastic state, allowing competence of cells to respond to signals prompting invasion or metastasis. In preliminary work in our laboratory, Brachyury knockdown by short hairpin RNA in oral cancer stem cells completely inhibited the EMT phenotype and cancer stem cell phenotypes (tumorigenicity and sphere formation) in vitro (Sugiura, unpublished data). If Brachyury directly regulates CSCs, Brachyury expression could be a predictor of the effects of chemotherapy and radiotherapy, because CSCs were shown to be resistant to chemotherapy and radiotherapy in vitro $(37,38)$. Furthermore, Brachyury could be a therapeutic target for anti-CSC therapy. Therefore, the effects of Brachyury in CSCs should be further investigated.

In conclusion, this study presents clinical evidence for an important role for Brachyury in EMT in oral SCC, and suggests that Brachyury expression and EMT patterns are useful prognostic markers.

\section{Acknowledgements}

This study was supported by Grants-in-Aid from the Ministry of Education, Culture, Sports, Science, and Technology of Japan (no. 23390465 to T. Sugiura).

\section{References}

1. Acloque H, Adams MS, Fishwick K, Bronner-Fraser M and Nieto MA: Epithelial-mesenchymal transitions: the importance of changing cell state in development and disease. J Clin Invest 119: $1438-1449,2009$
2. Kalluri R: EMT: when epithelial cells decide to become mesenchymal-like cells. J Clin Invest 119: 1417-1419, 2009.

3. Otsuki S, Inokuchi M, Enjoji M, et al: Vimentin expression is associated with decreased survival in gastric cancer. Oncol Rep 25: 1235-1242, 2011.

4. Kevans D, Wang LM, Sheahan K, et al: Epithelial-mesenchymal transition (EMT) protein expression in a cohort of stage II colorectal cancer patients with characterized tumor budding and mismatch repair protein status. Int J Surg Pathol 19: 751-760, 2011.

5. Soini Y, Tuhkanen H, Sironen R, et al: Transcription factors zeb1, twist and snail in breast carcinoma. BMC Cancer 11: 73, 2011.

6. van Nes JG, de Kruijf EM, Putter H, et al: Co-expression of SNAIL and TWIST determines prognosis in estrogen receptorpositive early breast cancer patients. Breast Cancer Res Treat 133: 49-59, 2011.

7. Sasaki K, Natsugoe S, Ishigami S, et al: Significance of Twist expression and its association with E-cadherin in esophageal squamous cell carcinoma. J Exp Clin Cancer Res 28: 158, 2009.

8. Nakashima H, Hashimoto N, Aoyama D, et al: Involvement of the transcription factor twist in phenotype alteration through epithelial-mesenchymal transition in lung cancer cells. Mol Carcinog 51: 400-410, 2011.

9. Yoshida S, Furukawa N, Haruta S, et al: Expression profiles of genes involved in poor prognosis of epithelial ovarian carcinoma: a review. Int J Gynecol Cancer 19: 992-997, 2009.

10. Yang TL, Wu CT, Ko JY, Wang CP, Lou PJ and Chang YL: Significance of tumor satellite variables in reflecting the epithelial-mesenchymal transition of tongue cancer. Oral Oncol 47: 720-724, 2011.

11. Wang C, Liu X, Huang H, et al: Deregulation of Snai2 is associated with metastasis and poor prognosis in tongue squamous cell carcinoma. Int J Cancer 130: 2249-2258, 2011.

12. Sakamoto K, Imanishi Y, Tomita T, et al: Overexpression of SIP1 and downregulation of E-cadherin predict delayed neck metastasis in stage I/II oral tongue squamous cell carcinoma after partial glossectomy. Ann Surg Oncol 19: 612-619, 2011.

13. Dang H, Ding W, Emerson D and Rountree CB: Snaill induces epithelial-to-mesenchymal transition and tumor initiating stem cell characteristics. BMC Cancer 11: 396, 2011.

14. Kojc N, Zidar N, Gale N, et al: Transcription factors Snail, Slug, Twist, and SIP1 in spindle cell carcinoma of the head and neck. Virchows Arch 454: 549-555, 2009.

15. Taube JH, Herschkowitz JI, Komurov K, et al: Core epithelial-tomesenchymal transition interactome gene-expression signature is associated with claudin-low and metaplastic breast cancer subtypes. Proc Natl Acad Sci USA 107: 15449-15454, 2011.

16. Yang MH, Hsu DS, Wang HW, et al: Bmil is essential in Twist1induced epithelial-mesenchymal transition. Nat Cell Biol 12: 982-992, 2011.

17. Karreth F and Tuveson DA: Twist induces an epithelial-mesenchymal transition to facilitate tumor metastasis. Cancer Biol Ther 3: 1058-1059, 2004.

18. Kispert A, Herrmann BG, Leptin M and Reuter R: Homologs of the mouse Brachyury gene are involved in the specification of posterior terminal structures in Drosophila, Tribolium, and Locusta. Genes Dev 8: 2137-2150, 1994.

19. Vidricaire G, Jardine K and McBurney MW: Expression of the Brachyury gene during mesoderm development in differentiating embryonal carcinoma cell cultures. Development 120: 115-122, 1994.

20. Fernando RI, Litzinger M, Trono P, Hamilton DH, Schlom J and Palena C: The T-box transcription factor Brachyury promotes epithelial-mesenchymal transition in human tumor cells. J Clin Invest 120: 533-544, 2010.

21. UICC: TNM Classification of Malignant Tumours. 7th edition. Wiley-Liss, New York, NY, 2009.

22. Wasi PN, Cohen B, Luthra UK and Torloni H: Histological typing of oral and oropharyngeal tumors. In: WHO: International Histological Classification of Tumors. WHO Press, Geneva, 1997.

23. Anneroth G, Batsakis J and Luna M: Review of the literature and a recommended system of malignancy grading in oral squamous cell carcinomas. Scand J Dent Res 95: 229-249, 1987.

24. Katoh M: Epithelial-mesenchymal transition in gastric cancer (Review). Int J Oncol 27: 1677-1683, 2005.

25. Schmalhofer O, Brabletz S and Brabletz T: E-cadherin, betacatenin, and ZEB1 in malignant progression of cancer. Cancer Metastasis Rev 28: 151-166, 2009.

26. Giehl K and Menke A: Microenvironmental regulation of E-cadherin-mediated adherens junctions. Front Biosci 13: 3975-3985, 2008. 
27. Satelli A and Li S: Vimentin in cancer and its potential as a molecular target for cancer therapy. Cell Mol Life Sci 68: 30333046, 2011.

28. Vuoriluoto K, Haugen H, Kiviluoto S, et al: Vimentin regulates EMT induction by Slug and oncogenic H-Ras and migration by governing Axl expression in breast cancer. Oncogene 30: 1436-1448, 2011.

29. Reya T, Morrison SJ, Clarke MF and Weissman IL: Stem cells, cancer, and cancer stem cells. Nature 414: 105-111, 2001.

30. Thiery JP: Epithelial-mesenchymal transitions in development and pathologies. Curr Opin Cell Biol 15: 740-746, 2003.

31. Mani SA, Guo W, Liao MJ, et al: The epithelial-mesenchymal transition generates cells with properties of stem cells. Cell 133: 704-715, 2008.

32. Blick T, Hugo H, Widodo E, et al: Epithelial mesenchymal transition traits in human breast cancer cell lines parallel the CD44(hi/)CD24 (lo/-) stem cell phenotype in human breast cancer. J Mammary Gland Biol Neoplasia 15: 235-252, 2010.

33. Du L, Yang Y, Xiao X, et al: Sox 2 nuclear expression is closely associated with poor prognosis in patients with histologically node-negative oral tongue squamous cell carcinoma. Oral Oncol 47: 709-713, 2011.
34. Episkopou V: SOX2 functions in adult neural stem cells. Trends Neurosci 28: 219-221, 2005

35. Fernando RI, Castillo MD, Litzinger M, Hamilton DH and Palena C: IL-8 signaling plays a critical role in the epithelialmesenchymal transition of human carcinoma cells. Cancer Res 71: 5296-5306, 2011

36. Sarkar D, Shields B, Davies ML, Muller J and Wakeman JA: Brachyury confers cancer stem cell characteristics on colorectal cancer cells. Int J Cancer 130: 328-337, 2012.

37. Ahmed N, Abubaker K, Findlay J and Quinn M: Epithelial mesenchymal transition and cancer stem cell-like phenotypes facilitate chemoresistance in recurrent ovarian cancer. Curr Cancer Drug Targets 10: 268-278, 2010

38. Alison MR, Lim SM and Nicholson LJ: Cancer stem cells: problems for therapy? J Pathol 223: 147-161, 2011. 